\begin{tabular}{|c|l|}
\hline Title & Hand-independent representation of tool-use pantomimes in the left anterior intraparietal cortex \\
\hline Author(s) & Ogawa, Kenji; Imai, Fumihito \\
\hline Citation & $\begin{array}{l}\text { Experimental brain research, 234(12),3677-3687 } \\
\text { https://doi.org/40.1007/300221-016-4765-7 }\end{array}$ \\
\hline Issue Date & 2016-12 \\
\hline Doc URL & http://hdl.handle.net/2115/67947 \\
\hline Rights & The final publication is available at link.springer.com \\
\hline Type & article (author version) \\
\hline File Information & ExpPantomime_Manuscript__HUSCAP..pdf \\
\hline
\end{tabular}

Instructions for use 


\title{
Hand-independent representation of tool-use pantomimes in the left anterior intraparietal cortex
}

\author{
Kenji Ogawa and Fumihito Imai \\ 5 Department of Psychology, Hokkaido University, Kita 10, Nishi 7, Kita-ku, Sapporo \\ 060-0810, Japan
}

Correspondence should be addressed to Dr. Kenji Ogawa, Department of Psychology,

Hokkaido University, Kita 10, Nishi 7, Kita-ku, Sapporo, 060-0810 Japan; Tel/Fax:

10 +81-011-706-4093; E-mail: ogawa@ let.hokudai.ac.jp. 


\begin{abstract}
Previous neuropsychological studies of ideomotor apraxia (IMA) indicated impairments in pantomime actions for tool use for both right and left hands following lesions of parieto-premotor cortices in the left hemisphere. Using functional magnetic resonance imaging (fMRI) with multi-voxel pattern analysis (MVPA), we tested the hypothesis that the left parieto-premotor cortices are involved in the storage or retrieval of hand-independent representation of tool use actions. In the fMRI scanner, one of three kinds of tools was displayed in pictures or letters, and the participants made pantomimes of the use of these tools using the right hand for the picture stimuli or with the left hand for the letters. We then used MVPA to classify which kind of tool the subjects were pantomiming. Whole-brain searchlight analysis revealed successful decoding using the activities largely in the contralateral primary sensorimotor region, ipsilateral cerebellum, and bilateral early visual area, which may reflect differences in low-level sensorimotor components for three types of pantomimes. Furthermore, a successful cross-classification between the right and left hands was possible using the activities of the left inferior parietal lobule (IPL) near the junction of the anterior intraparietal sulcus. Our finding indicates that the left anterior intraparietal cortex plays an important role in the production of tool-use pantomimes in a hand-independent manner, and independent of stimuli modality.
\end{abstract}

\title{
Keywords
}

fMRI; multi-voxel pattern analysis; tool use; pantomime; anterior intraparietal cortex 


\section{Introduction}

Previous studies have shown that the parieto-premotor cortices in the left hemisphere

5 play important roles in the production of tool-related actions (Johnson-Frey 2004; Lewis 2006). Neuropsychological studies have indicated that lesions of the left posterior parietal cortex (PPC), mainly in the inferior parietal lobule (IPL) or supramarginal gyrus, including the anterior intraparietal cortex, as well as those of the inferior frontal gyrus cause ideomotor apraxia (IMA), which accompanies impaired tool-use pantomimes for both right and left hands (Heilman et al. 1982; Donkervoort et al. 2000; Haaland et al. 2000; Buxbaum 2001; Goldenberg et al. 2007). IMA is neither caused by deficits in basic motor or sensory processing nor by general cognitive impairments, but rather by deficits in more abstract or higher level of motor engram, independent of the hand used (Goldenberg 2009). In that sense, IMA contrasts with limb-kinetic apraxia due to the impaired formation of 15 innervatory motor patterns, which is usually unilaterally affected to the effector contralateral to the damaged site in the primary sensorimotor cortex (Denes et al. 1998).

Consistent with these lesion studies, previous neuroimaging studies using functional magnetic resonance imaging (fMRI) or positron emission tomography (PET) have shown that PPC as well as premotor cortex in the left hemisphere is activated by the pantomiming of tool use (Choi et al. 2001; Rumiati et al. 2004; Fridman et al. 2005; $\underline{\text { Hermsdorfer et al. }}$ 2007; Imazu et al. 2007; Vingerhoets et al. 2011) and tool-use imagery (Imazu et al. 2007;

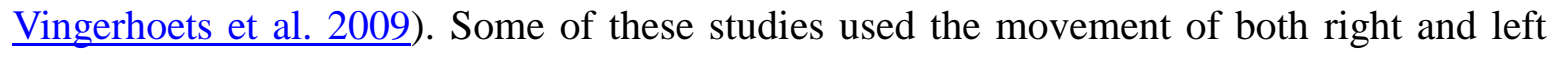
hands, and they revealed that the left-lateralized PPC and premotor network are activated for the pantomiming of both dominant and non-dominant hands (Moll et al. 2000; Johnson 
and Grafton 2003; Johnson-Frey et al. 2005). Viewing or naming of tools without motor execution also activates the left PPC, mainly in the IPL, and premotor cortex, together with the regions representing specific object categories in the temporal cortex (Chao et al. 1999; Chao and Martin 2000; Noppeney et al. 2006; Mahon et al. 2007; Mruczek et al. 2013;

5 Peeters et al. 2013). The IPL has been repeatedly shown to be integral to the knowledge on skilled tool-use or object manipulation (Kellenbach et al. 2003; Boronat et al. 2005; Ishibashi et al. 2011) with distinct functional connectivities with the premotor and temporal regions (Garcea and Mahon 2014). A right visual field advantage for the visual processing of tools (Garcea et al. 2012) and a tool-selective response in the left IPL independent of visual field were reported (Garcea et al. 2016), which is consistent with left hemisphere dominance for tool-selective processing. Recent studies using anodal transcranial direct current stimulation (tDCS) showed that electrical stimulation of the left PPC improves the processing of gesture or imitation in healthy participants (Weiss et al. 2013) and also in IMA patients (Bianchi et al. 2015; Bolognini et al. 2015), which further suggest the importance of left PPC for gesture processing.

Among the dorsal visual stream within the PPC, the dorso-dorsal pathway comprising the superior parietal lobule (SPL) and ventro-dorsal pathway, including the IPL, are distinct pathways (Rizzolatti and Matelli 2003; Binkofski and Buxbaum 2013). Online sensorimotor control with objects based on their perceptual attributes ("acting on") is mainly related to the SPL, whereas the stored knowledge of tool-use ("acting with") is subserved by the IPL (Johnson and Grafton 2003; Brandi et al. 2014). In addition, the interaction between dorsal and ventral stream processing for the integration of proper tool-use knowledge occurs within the IPL (Almeida et al. 2013; Mahon et al. 2013; 


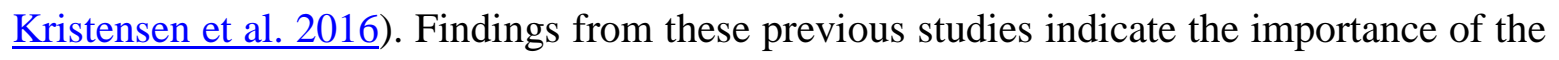
IPL in skilled tool-use.

One prominent feature of IMA is that unilateral damage predominantly in the left hemisphere causes deficits in both sides of the body (Goldenberg 2009). In this study, we

5 tested the hypothesis that the left parieto-premotor cortices are related to the retrieval or storage of neural representations for tool use, which is independent of the hand used. To test this prediction, we used fMRI with multi-voxel pattern analysis (MVPA) or decoding analysis to classify pantomime actions. Compared with a conventional mass univariate analysis, MVPA focuses on differences in the spatial activation patterns of multiple voxels (Norman et al. 2006) and can be used to reveal specific neural representations (Haynes and

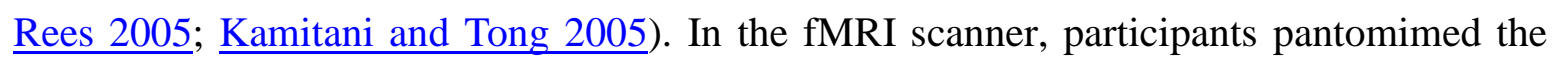
use of tools with their right or left hand. We then used MVPA to decode which tool the subjects were pantomiming in a hand-invariant manner based on the classifier's generalization ability between the right and left hands. Different types of the stimulus

15 presentation (pictures and letters of tools for right- and left-hand conditions, respectively) were used to exclude confounding effects in visual display; the possibility that the successful hand-invariant cross-decoding could be due to that the subjects are observing the same visual stimulus (pictures or letters). In the same manner, the successful hand-invariant classification was not due to differences in low-level motor output or proprioceptive inputs

20 because those are different between the right and left hands. A successful hand-independent "cross-classification" (Kaplan et al. 2015) would thus indicate the common neural representation of tool use for both hands. 


\section{Materials and Methods}

\subsection{Subjects}

Subjects included 11 volunteers (6 males and 5 females) with a mean age of 25.6 years (range, 20-36 years). All participants were right handed as assessed by a modified version of the Edinburgh Handedness Inventory (일ield 1971) for Japanese participants (ㅂatta and Nakatsuka 1975). Written informed consent was obtained from all participants in accordance with the Declaration of Helsinki. The experimental protocol received approval from the local ethics committee.

\subsection{Task procedures}

The participants made pantomimes of the use of three types of tools. Within each task block of $12 \mathrm{~s}$, the grayscale pictures or written letters of one of the three tools (scissors, hammer, or key) written in Japanese characters were presented to the participants for $1 \mathrm{~s}$, which was interleaved with fixation for 1s (Fig. 1). Throughout the study, the picture and letter stimuli were always associated with the right and left hand conditions, respectively. For the right hand condition, only one picture was used for each tool and in all three pictures, the handle (or graspable part of the tool) was oriented toward right (Fig. 1A). Different types of the stimulus presentation were used to exclude the possibility that the successful hand-invariant cross-classification is due to visual confounds. A single block contained the same type of tool. The participants were asked to make pantomimes of the use of the displayed tool once every 2 s, immediately following each stimulus display (Fig. 1B). Participants thus performed the same pantomime 6 times per block. A fixation cross was displayed for $12 \mathrm{~s}$ 
during interblock intervals. The participants underwent a total of 8 sessions ( 4 each for the right and left hands), with each session comprising 12 blocks. One session lasted for approximately $5 \mathrm{~min}$. The right- or left-hand session order was counterbalanced as A-B-B-A-A-B-B-A with $\mathrm{A}$ and $\mathrm{B}$ pseudo-randomly assigned to the right hand (with

5 pictures) and left hand (with letters) conditions. The participants' hand movements were monitored online as well as videotaped and were checked for their compliance.

FIGURE 1 ABOUT HERE

Stimuli were presented on a liquid crystal display and projected onto a custom-made viewing screen. The participants were in the supine position in the scanner and viewed the screen via a mirror, and they were unable to see their hand throughout this task. The participants' heads were immobilized with foam pads to minimize head movements. The maximum amount of movement in the participants' heads, which was estimated by aligning the functional images (see below), ranged from $0.18 \mathrm{~mm}$ to $1.54 \mathrm{~mm}$, with a median of 0.39 mm within a session.

\subsection{MRI acquisition}

All scans were performed on a Siemens (Erlangen, Germany) 3-Tesla Prisma scanner with a 20-channel head coil at the Research and Education Center for Brain Science, Hokkaido University. T2*-weighted echo planar imaging (EPI) was used to acquire a total of 99 scans each session, with a gradient echo EPI sequence. The first three scans of each session were discarded to allow for T1 equilibration. The scanning parameters were repetition time (TR), 
3,000 ms; echo time (TE), $30 \mathrm{~ms}$; flip angle (FA), 90; field of view (FOV), $192 \times 192 \mathrm{~mm}$; matrix, $94 \times 94 ; 36$ axial slices; and slice thickness, $3.0 \mathrm{~mm}$ with $0.75 \mathrm{~mm}$ gap. T1-weighted anatomical imaging with an MP-RAGE sequence was performed with the following parameters: TR, 2,300 ms; TE, $2.32 \mathrm{~ms}$; FA, 8; FOV, $256 \times 256 \mathrm{~mm}$; matrix,

$5 \quad 256 \times 256$; 192 axial slices; and slice thickness, $1 \mathrm{~mm}$ without gap.

\subsection{Processing of fMRI data}

Image preprocessing was performed using the SPM12 software (Wellcome Department of Cognitive Neurology, http://www.fil.ion.ucl.ac.uk/spm). All functional images were first

10 realigned to adjust for motion-related artifacts. The realigned images were then spatially normalized with the Montreal Neurological Institute (MNI) template based on the affine and non-linear registration of co-registered T1-weighted anatomical images (normalization procedure of SPM) and were resampled into 3-mm-cube voxels with sinc interpolation. All images were spatially smoothed using a Gaussian kernel of $6 \times 6 \times 6-\mathrm{mm}$ full width at half-maximum. Smoothing was only performed in the mass univariate analysis (see below) as this could blur fine-grained information contained in the multi-voxel activity (Mur et al. 2009).

Using the general linear model (GLM), the 12 blocks per session were modeled as separate 12 box-car regressors that were convolved with a canonical hemodynamic response function. Low-frequency noise was removed using a high-pass filter with a cut-off period of $128 \mathrm{~s}$, and serial correlations among scans were estimated with an autoregressive model implemented in SPM12. This analysis yielded 12 independently estimated 
parameters (beta-values) per session for each individual voxel, which were subsequently used as inputs for MVPA. We also used $t$-values (Misaki et al. 2010) instead of beta-values for MVPA, which yielded similar results.

5

\subsection{MVPA}

The multivariate classification analysis of fMRI was performed with a multi-class classifier based on a linear support vector machine (SVM) implemented in LIBSVM (http://www.csie.ntu.edu.tw/ cjlin/libsvm/) with default parameters (a fixed regularization parameter $\mathrm{C}=1$ ). Multi-class classification, implemented in LIBSVM, was used to classify the three types of pantomime using the "one-against-one" method in which all pairwise classifiers among the tools were conducted and then each output was compared to produce the label with maximal probability. Parameter estimates (beta-values) of each trial of voxels 
within regions of interest were used as inputs to the classifier.

We conducted a volume-based "searchlight" analysis (Kriegeskorte et al. 2006). Classification was performed using multi-voxel activation patterns within a 6 -mm-radius sphere (searchlight) that contained 33 voxels. The searchlight moved over the gray matter

5 of the whole brain, and the average classification accuracy for each searchlight with leave-one-session-out cross-validation was assigned to the sphere's center voxel. The resulting map of the decoding accuracy was averaged over the participants.

We conducted two types of classification analysis. First, we ran a hand-dependent analysis, which classified the three types of pantomime actions, separately for each condition, i.e. left hand with letters vs. right hand with pictures. Second, hand-independent analysis was performed with cross-classification between the left hand with letters and right hand with pictures conditions. In this analysis, the classifier was first trained to discriminate the three types of pantomimes made with the right hand. The same decoder was then tested to see if it could classify pantomimes with the left hand. We also conducted classification in the reverse direction: trained with the left hand and tested with the right hand. Such a cross-conditional MVPA (Ogawa and Inui 2012; Ogawa and Imamizu 2013; Wurm and Lingnau 2015) or cross-classification, a cross-validation between trials with different sets of tasks or stimuli, has been previously used to investigate the similarity or invariance of neural representations by testing the generalization of a classifier between different conditions or modalities (Kaplan et al. 2015). A one-sample $t$-test was used to determine whether the observed decoding accuracy was significantly higher than chance $(33.3 \%)$ with intersubject difference treated as a random factor (degrees of freedom $=10$ ). We used a voxel-level threshold of $p<0.001$ for the hand-independent cross-classification analysis, 
and a more liberal threshold of $p<0.005$ for the hand-dependent analysis to increase statistical power because the sample size of the hand-dependent analysis was half that of the cross-classification analysis. To control the problem of multiple comparisons, we also applied a cluster-level FWE threshold of $p<0.05$ for both analyses.

\subsection{Additional experiment with simple manual movements}

To confirm that the successful between hands cross-classification was specific to tool use actions rather than to low-level visuomotor processes, an additional fMRI experiment with simple manual movements was conducted. Subjects included 11 volunteers (all right-handed males) with a mean age of 29.5 years (range, 23-37 years), and five subjects participated in the previous pantomime experiment. The maximum movement in the participants' heads, estimated by aligning the functional images, ranged from $0.21 \mathrm{~mm}$ to $0.71 \mathrm{~mm}$, with a median of $0.40 \mathrm{~mm}$ within a session.

In this experiment, participants performed individual finger-tapping of four digits

15 (index, middle, ring, and little fingers), which was visually cued by a single Japanese character. During task blocks of 12 iterations, the number "12" was displayed and then counted down for every $1 \mathrm{~s}$ until " 1 " was displayed. Synchronized with the timing of this visual cue, the subjects made repeated tapping movements with the same finger 12 times, once for every second. A fixation cross was displayed for $12 \mathrm{~s}$ during interblock intervals. The participants underwent a total of 8 sessions (4 each for the right and left hands), with each session comprising 12 blocks. One session lasted approximately 5 min. The number of trials and sessions per subject was the same as those in the pantomime experiment. The same preprocessing procedures were applied to fMRI data and MVPA to classify which 
finger the subjects were tapping with, as in the pantomime decoding. We hypothesized that if low-level visuomotor processes had contributed to the successful classification of pantomimes, significant classification accuracy could also be obtained in this control experiment. 


\section{Results}

\subsection{Mass univariate analysis}

We analyzed the activated regions of the brain using conventional univariate analysis of single voxels. First, we analyzed the areas that were significantly activated during 5 pantomimes, collapsed across both three types of pantomimes and the left and right hands, compared with the regions that were activated at rest. The regions largely included the primary sensorimotor cortex, medial motor regions including the supplementary motor area, posterior parietal cortex, cerebellum, and early visual cortex (Fig. 2A and Table 1). Next, by directly comparing the left hand with letters and right hand with pictures conditions, we found activations mostly in the sensorimotor cortex contralateral to the hand used, together with the ipsilateral cerebellum (Fig. 2B and Table 2). We also found greater activations in the early visual areas in the right-hand condition, which was triggered by the pictorial display of tools, compared with that in the left-hand condition, which was triggered by letters.

\subsection{MVPA}

We first ran hand-dependent classification analysis, which decoded the three types of pantomime actions separately for the right and left hand trials. The analysis revealed significantly above-chance decoding accuracy largely in the contralateral primary sensorimotor region as well as in the ipsilateral cerebellum (Fig. 3 and Table 3). Compared 
with at rest, we found that voxels in the early visual cortex were significantly more active in both the right- and left-hand conditions, and the cluster size was greater in the right- than in the left-hand condition. The average classification accuracies of the peak voxels were $56.3 \%$ and $49.2 \%$ for the right- and left-hand condition, respectively.

Next, we performed hand-independent analysis with cross-classification between the right and left hands. In this analysis, the classifier was first trained to discriminate the three pantomimes performed with the right hand. The same decoder was then tested to see if it could classify pantomimes with the left hand. We also conducted classification in the reverse direction, i.e., trained with the left hand and tested with the right hand. A successful cross-classification between the right and left hands was possible with the left IPL near the junction of the anterior intraparietal sulcus (aIPS) and post-central sulcus, with a peak mean classification accuracy of $43.1 \%$ (Fig. 4 and Table 3).

FIGURES 3, 4 \& TABLE 3 ABOUT HERE

\subsection{MVPA with simple manual movements}

The searchlight analysis of tapping fMRI data was separately performed for each hand, as for the pantomime decoding. This revealed that a large cluster including the sensorimotor cortex, contralateral to the hand, showed a significant decoding accuracy for the finger tapping (peak voxel coordinate of $([-33,-22,47], t(10)=8.50)$ and left hand $([42,-16,53]$, $t(10)=9.41)$ with a voxel-level uncorrected threshold of $p<0.005$ with a cluster-level FWE threshold of $p<0.05$, which is consistent with results for the pantomime decoding (Fig. 5). A searchlight analysis of cross-classification between hands was then conducted. 
No significant voxel was found, even with a more lenient threshold (voxel-level uncorrected threshold of $p<0.01$ with a cluster-level FWE threshold of $p<0.05$ ) than that used in the pantomime experiment.

Then, the region of interest (ROI)-based MVPA was conducted for further

5 comparisons between the pantomime and tapping experiments. To avoid circular analysis (Kriegeskorte et al. 2009), ROIs were defined in the bilateral aIPS as $10 \mathrm{~mm}$-radius spheres centered on the mean coordinates of the meta-analysis of independent human neuroimaging studies for manual actions (Talairach coordinates of $[-40,-39,43]$ for the left and $[41,-40$, 45] for the right hemispheres, respectively, which were converted into MNI coordinates; http://imaging.mrc-cbu.cam.ac.uk/imaging/MniTalairach) (Tunik et al. 2007). For pantomime decoding, significant accuracy was again found in the left aIPS $(t(10)=3.23, p$ $<0.01)$ but not in the right aIPS $(t(10)=0.86, p=0.41)$, replicating the searchlight results. In contrast, no significant classification accuracy was found for the tapping in either the left aIPS $(t(10)=-0.39, p=0.71)$ or the right aIPS $(t(10)=0.49, p=0.64)$. These results indicate that the successful cross-classification of the pantomime cannot be attributed to the differences in low-level motor processing.

Finally, the same MVPA was conducted on a control ROI, a 10-mm radius sphere centered on $[0,0,0]$ mostly covering the white matter, and this analysis showed no significant decoding accuracy for either pantomime $(t(10)=-0.94, p=0.37)$ or tapping $20 \quad(t(10)=-0.70, p=0.50)$. This result supports the hypothesis that successful decoding was not due to spurious factors such as task-related head or arm movements. 


\section{Discussion}

In this study, we used fMRI with MVPA to reveal a hand-independent neural substrate for tool use. First, when we independently used the right and left hand datasets to classify pantomimes, the regions with significantly above-chance accuracy largely included the primary sensorimotor areas contralateral to the hand together with the ipsilateral cerebellum. The regions also included the early visual cortex. These results reflect low-level differences in the visual inputs of stimulus images or movement kinematics represented in the early sensorimotor areas. Furthermore, using MVPA with cross-classification between the right and left hands, we showed successful classification only in the left IPL. Additional control experiment using simple manual tapping confirmed that the successful between-hands classification is not due to low-level visuomotor processing. In this case, between-hand classification for finger tapping was not possible with both the whole-brain searchlight and the ROI analysis on IPL. However, we observed significantly above-chance accuracy under within-hand decoding analysis in the sensorimotor region contralateral to the hand, which is consistent with the pantomime decoding results. This indicates that the left IPL is not related to simple low-level manual movements for both hands. Our finding thus indicates that the left IPL is related to the production of tool-use pantomimes in a hand-independent manner.

Our experimental design has two potential limitations. First, the participants always used their right and left hands to pantomime the use of tools given as pictures and words, respectively. Thus, the difference between right versus left hand usage during pantomime is confounded by different types of stimuli presented for each hand, which may have led to 
the distinct activations, primarily in the visual cortex together with differences in the PPC, associated with pantomimes using the right hand for the pictures versus left hand for the letters in this study (Fig. $2 \& 3$ ). Previous studies have reported that viewing both the picture and word of tools commonly activated the left aIPS (Noppeney et al. 2006) and the IPL but with a greater activation response to the pictures than to the words (Boronat et al. 2005) without pantomiming or actual tool use. These results suggest that the left aIPS revealed by our cross-classification could also reflect the response to viewing of pictures or letters of the tools. Irrespectively, the goal of this paper is to look for shared representations of tool pantomimes irrespective of hand usage (and/or type of stimuli used). Successful hand/stimuli-independent cross-classification within IPL would indicate common neural representations of tool use for both hands, and independent of stimuli modality. The second limitation of this study comes from the fact that we used only one picture for each tool and in all three pictures the handle (graspable part) was oriented toward the right (Fig. 1A). Because the picture presentation was always associated with the right hand condition, the participants made pantomimes with the graspable parts of each tool located toward their dominant right hand. Having the viewing handle directed toward the acting hand is known to automatically potentiate the action representation in the viewer via object affordance (Tucker and Ellis 1998), which could be a potential confound in the current study. However, previous fMRI studies have shown that viewing objects in different orientations affects activity in the occipito-parietal junction in the right hemisphere (Valyear et al. 2006; Rice et al. 2007) and in the bilateral occipital cortex (Macdonald and Culham 2015) but not in the left anterior intraparietal cortex, which indicates that tool orientation was unlikely to affect the current finding. 
In previous neuroimaging studies, researchers asked participants to make pantomimes with both right and left hands and showed that the left-lateralized parietal and premotor networks are activated for both dominant and non-dominant hands (Moll et al. 2000; Johnson and Grafton 2003; Johnson-Frey et al. 2005). However, with a conventional subtraction method based on mass univariate analysis, it cannot be decided whether there is a common neural representation for both hands, or separate representations for each hand contained within the same region. Using MVPA to analyze differences in the patterns of multi-voxel activities together with cross-classification between hands, the current study successfully showed the common neural representation for both hands. Our findings thus support the existence of the effector-invariant movement representation of tool use in the left IPL, which is consistent with the lesion cases of IMA (Buxbaum et al. 2007; Binkofski and Buxbaum 2013). Notably, the observed accuracy for the cross-classification (43.1\%) is significantly higher than chance $(33 \%)$ but is still relatively low. Thus, our successful cross-classification does not exclude the presence of distinct neural representation for the right and left hands, which could be intermingled with the shared substrates within the left IPL.

The left IPL observed in this study was located close to aIPS, which is considered to be a human homolog of AIP in non-human primates (Grefkes and Fink 2005; $\underline{\text { Tunik et al. }}$ 2007). aIPS was originally known as a cross-modal area between visual and proprioceptive inputs for object-directed manual actions revealed in monkeys (Murata et al. 2000) as well as in a human neuroimaging study (Grefkes et al. 2002). aIPS is responsive to skilled functional usage of tools more than to inherent graspability of objects (Valyear et al. 2007; Vingerhoets et al. 2009). This area is also responsive to manual actions that require both 
hands and tools (Jacobs et al. 2010), which indicates the existence of an effector-independent representation of skilled manual movement in aIPS. Recent MVPA study showed similar activation patterns between viewing tools and hands in left IPS, which indicates that shared neural substrates for object manipulation in this area (Bracci et

5 al. 2016). Another recent study used MVPA to decode pantomined actions perfomed with the right hand and found that left IPL activation could be used to classify pantomimes across different tools having similar actions (e.g., scissors and pliers) (hen et al. 2016). aIPS is also related to a multi-modal or abstract action representation revealed by recent MVPA studies (Ogawa and Inui 2011; Oosterhof et al. 2012). Furthermore, the anterior part of IPL in the left hemisphere is related to encoding action goals (Hamilton and Grafton 2006) or tool-related action outcomes (Leshinskaya and Caramazza 2015). These previous neuroimaging studies indicate that IPL contains an abstract level of action representation rather than low-level movement parameters, which is consistent with the current findings. Our study further extends previous findings by showing successful generalization of 15 pantomine decoding across the right and left hands.

A recent study used MVPA to decode an effector-independent representation using two types of simple object-directed actions: grasping or reaching (Gallivan et al. 2013). Using the same cross-classification technique that we used in the present study they found that PPC and the ventral premotor cortex are related to movement plans for both right and left 20 hands, which is generally consistent with our results. The difference between their study results and the current results is that they showed almost an equal importance in the classification accuracy of bilateral PPC, whereas our results showed left-hemispheric dominance. Considering that a number of previous neuroimaging studies on tool use (Moll 
et al. 2000; Choi et al. 2001; Rumiati et al. 2004; Fridman et al. 2005; Johnson-Frey et al. 2005; Imazu et al. 2007) also showed left-hemisphere dominance, this discrepancy may be a result of the movement types. The difference in neural substrates between simple manual actions and tool use is also supported by the fact that IMA patients showed no impairments in visually guided grasping or reaching ( failed to show successful decoding in PPC with non-tool manual actions such as both visually guided tracking (ㅁgawa and Imamizu 2013) and reaching (Kim et al. 2015), which may further indicate differences in neural substrates between tool-use and non-tool-use actions.

We further conducted a control experiment using simple manual movements and confirmed that the successful between-hands classification is not due to low-level visuomotor processing. However, our current experiment still cannot determine whether our result is specific to tool-use and not to non-tool actions with objects, such as grasping or manipulation of an object, as well as intransitive non-object related gestures. Thus, the current study cannot determine whether the left aIPS is specific to acquired functional use of familiar tools rather than processing of the inherent affordance (graspability) of objects or complex manual actions in general. Impairments in production and imitation of intransitive gestures is also known in IMA, and therefore, further studies with additional experiments are needed to clarify whether the cross-decoding in the left IPL is possible for gesture production. Another limitation of the current study is that the lack of significance in decoding accuracy does not conclusively demonstrate that the neurons in a local region are totally unselective to experimental manipulations. The decoding accuracy of MVPA may be determined by a sampling bias caused by spatial patterns of distinct neuronal 
populations accompanying vasculature units as well as the spatial resolution of fMRI (Bartels et al. 2008; Gardner 2010). It is thus still possible that MVPA cannot detect small differences in the spatial activation patterns caused by the underlying neural representation in the fMRI data.

The neural substrates responsible for the IMA, particularly with impairments in tool-use pantomimes, have been controversial, with inconsistent reports. One traditional view is that the IMA is caused by a posterior parietal lesion in the dominant hemisphere (Heilman et al. 1982; Buxbaum 2001). In contrast, a study using voxel-based lesion mapping showed that the pantomime disturbance is more related to the inferior frontal cortex rather to the IPL (Goldenberg et al. 2007; Goldenberg 2009). The present study does not exclude the importance of the inferior frontal region, for which MVPA may not be sensitive enough to reveal neural representations, as was previously discussed as the limitation of this method, or due to a lack of statistical power because of limited number of participants in the current study. Another possibility is that the pantomime is subserved by a widely distributed network in the left hemisphere, including premotor and parietal cortices together with connecting fiber tracts, which could not be analyzed by MVPA. More recent studies with a larger sample of acute stroke patients showed that the IPL is responsible for pantomime integrity (Hoeren et al. 2014; Martin et al. 2015; Martin et al. 2016), which is in accordance with the present finding.

In summary, using fMRI cross-conditional MVPA between the right and left hands, we revealed a hand-independent movement representation for tool use in the left IPL. Our findings are consistent with those of previous lesion studies with IMA and provide an insight into how our skillful motor knowledge or engrams are stored and retrieved in our 
brain.

Acknowledgements This work was supported in part by a Grant-in-Aid for Exploratory Research (No. 26540059) to K.O. 


\section{References}

Almeida J, Fintzi AR, Mahon BZ (2013) Tool manipulation knowledge is retrieved by way of the ventral visual object processing pathway. Cortex 49:2334-2344

Bartels A, Logothetis NK, Moutoussis K (2008) fMRI and its interpretations: an illustration on directional selectivity in area V5/MT. Trends in Neurosciences 31:444-453

Bianchi M, Cosseddu M, Cotelli M, et al. (2015) Left parietal cortex transcranial direct current stimulation enhances gesture processing in corticobasal syndrome. Eur $\mathbf{J}$ Neurol 22:1317-1322

Binkofski F, Buxbaum LJ (2013) Two action systems in the human brain. Brain Lang $127: 222-229$

Bolognini N, Convento S, Banco E, Mattioli F, Tesio L, Vallar G (2015) Improving ideomotor limb apraxia by electrical stimulation of the left posterior parietal cortex. Brain 138:428-439

Boronat CB, Buxbaum LJ, Coslett HB, Tang K, Saffran EM, Kimberg DY, Detre JA (2005) Distinctions between manipulation and function knowledge of objects: evidence from functional magnetic resonance imaging. Brain Res Cogn Brain Res $23: 361-373$

Bracci S, Cavina-Pratesi C, Connolly JD, Ietswaart M (2016) Representational content of occipitotemporal and parietal tool areas. Neuropsychologia 84:81-88

Brandi ML, Wohlschlager A, Sorg C, Hermsdorfer J (2014) The neural correlates of planning and executing actual tool use. J Neurosci 34:13183-13194

Buxbaum LJ (2001) Ideomotor apraxia: a call to action. Neurocase 7:445-458

Buxbaum LJ, Kyle K, Grossman M, Coslett HB (2007) Left inferior parietal 
representations for skilled hand-object interactions: evidence from stroke and corticobasal degeneration. Cortex 43:411-423

Chao LL, Haxby JV, Martin A (1999) Attribute-based neural substrates in temporal cortex for perceiving and knowing about objects. Nat Neurosci 2:913-919

5 Chao LL, Martin A (2000) Representation of manipulable man-made objects in the dorsal stream. Neuroimage 12:478-484

Chen Q, Garcea FE, Mahon BZ (2016) The Representation of Object-Directed Action and Function Knowledge in the Human Brain. Cereb Cortex 26:1609-1618

Choi SH, Na DL, Kang E, Lee KM, Lee SW, Na DG (2001) Functional magnetic resonance imaging during pantomiming tool-use gestures. Exp Brain Res 139:311-317

Denes G, Mantovan MC, Gallana A, Cappelletti JY (1998) Limb-kinetic apraxia. Mov Disord 13:468-476

Donkervoort M, Dekker J, van den Ende E, Stehmann-Saris JC, Deelman BG (2000) Prevalence of apraxia among patients with a first left hemisphere stroke in rehabilitation centres and nursing homes. Clin Rehabil 14:130-136

Fridman EA, Immisch I, Hanakawa T, et al. (2005) The role of the dorsal stream for gesture production. Neuroimage 29:417-428

Gallivan JP, McLean DA, Flanagan JR, Culham JC (2013) Where one hand meets the other: limb-specific and action-dependent movement plans decoded from preparatory signals in single human frontoparietal brain areas. J Neurosci 33:1991-2008

Garcea FE, Almeida J, Mahon BZ (2012) A right visual field advantage for visual processing of manipulable objects. Cogn Affect Behav Neurosci 12:813-825 
Garcea FE, Kristensen S, Almeida J, Mahon BZ (2016) Resilience to the contralateral visual field bias as a window into object representations. Cortex 81:14-23

Garcea FE, Mahon BZ (2014) Parcellation of left parietal tool representations by functional connectivity. Neuropsychologia 60:131-143

5 Gardner JL (2010) Is cortical vasculature functionally organized? Neuroimage 49:1953-1956

Goldenberg G (2009) Apraxia and the parietal lobes. Neuropsychologia 47:1449-1459

Goldenberg G, Hermsdorfer J, Glindemann R, Rorden C, Karnath HO (2007) Pantomime of Tool Use Depends on Integrity of Left Inferior Frontal Cortex. Cereb Cortex $17: 2769-2776$

Grefkes C, Fink GR (2005) The functional organization of the intraparietal sulcus in humans and monkeys. J Anat 207:3-17

Grefkes C, Weiss PH, Zilles K, Fink GR (2002) Crossmodal processing of object features in human anterior intraparietal cortex: an fMRI study implies equivalencies between humans and monkeys. Neuron 35:173-184

Haaland KY, Harrington DL, Knight RT (1999) Spatial deficits in ideomotor limb apraxia. A kinematic analysis of aiming movements. Brain 122 ( Pt 6):1169-1182

Haaland KY, Harrington DL, Knight RT (2000) Neural representations of skilled movement. Brain 123 ( Pt 11):2306-2313

Hamilton AF, Grafton ST (2006) Goal representation in human anterior intraparietal sulcus. J Neurosci 26:1133-1137

Hatta T, Nakatsuka Z (1975) Handedness inventry. In: Ohno D (ed) Papers on Celebrating 63rd Birthday of Prof. Ohnishi. Osaka City University, Osaka, Japan, pp 224-245 
Haynes JD, Rees G (2005) Predicting the orientation of invisible stimuli from activity in human primary visual cortex. Nat Neurosci 8:686-691

Heilman KM, Rothi LJ, Valenstein E (1982) Two forms of ideomotor apraxia. Neurology $32: 342-346$

5 Hermsdorfer J, Terlinden G, Muhlau M, Goldenberg G, Wohlschlager AM (2007) Neural representations of pantomimed and actual tool use: evidence from an event-related fMRI study. Neuroimage 36 Suppl 2:T109-118

Hoeren M, Kummerer D, Bormann T, et al. (2014) Neural bases of imitation and pantomime in acute stroke patients: distinct streams for praxis. Brain $137: 2796-2810$

Imazu S, Sugio T, Tanaka S, Inui T (2007) Differences between actual and imagined usage of chopsticks: an fMRI study. Cortex 43:301-307

Ishibashi R, Lambon Ralph MA, Saito S, Pobric G (2011) Different roles of lateral anterior temporal lobe and inferior parietal lobule in coding function and manipulation tool knowledge: evidence from an rTMS study. Neuropsychologia 49:1128-1135

Jacobs S, Danielmeier C, Frey SH (2010) Human anterior intraparietal and ventral premotor cortices support representations of grasping with the hand or a novel tool. Journal of Cognitive Neuroscience 22:2594-2608

Johnson SH, Grafton ST (2003) From 'acting on' to 'acting with': the functional anatomy of object-oriented action schemata. Progress in Brain Research 142:127-139

Johnson-Frey SH (2004) The neural bases of complex tool use in humans. Trends Cogn Sci 8:71-78

Johnson-Frey SH, Newman-Norlund R, Grafton ST (2005) A distributed left hemisphere 
network active during planning of everyday tool use skills. Cereb Cortex $15: 681-695$

Kamitani Y, Tong F (2005) Decoding the visual and subjective contents of the human brain. Nat Neurosci 8:679-685

5 Kaplan JT, Man K, Greening SG (2015) Multivariate cross-classification: applying machine learning techniques to characterize abstraction in neural representations. Front Hum Neurosci 9:151

Kellenbach ML, Brett M, Patterson K (2003) Actions speak louder than functions: the importance of manipulability and action in tool representation. J Cogn Neurosci $15: 30-46$

Kim S, Ogawa K, Lv J, Schweighofer N, Imamizu H (2015) Neural Substrates Related to Motor Memory with Multiple Timescales in Sensorimotor Adaptation. PLoS Biol 13:e1002312

Kriegeskorte N, Goebel R, Bandettini P (2006) Information-based functional brain mapping. Proc Natl Acad Sci U S A 103:3863-3868

Kriegeskorte N, Simmons WK, Bellgowan PS, Baker CI (2009) Circular analysis in systems neuroscience: the dangers of double dipping. Nature Neuroscience $12: 535-540$

Kristensen S, Garcea FE, Mahon BZ, Almeida J (2016) Temporal Frequency Tuning Reveals Interactions between the Dorsal and Ventral Visual Streams. J Cogn Neurosci 28:1295-1302

Leshinskaya A, Caramazza A (2015) Abstract categories of functions in anterior parietal lobe. Neuropsychologia 76:27-40 
Lewis JW (2006) Cortical networks related to human use of tools. Neuroscientist $12: 211-231$

Macdonald SN, Culham JC (2015) Do human brain areas involved in visuomotor actions show a preference for real tools over visually similar non-tools? Neuropsychologia 77:35-41

Mahon BZ, Kumar N, Almeida J (2013) Spatial frequency tuning reveals interactions between the dorsal and ventral visual systems. J Cogn Neurosci 25:862-871

Mahon BZ, Milleville SC, Negri GA, Rumiati RI, Caramazza A, Martin A (2007) Action-related properties shape object representations in the ventral stream. Neuron $55: 507-520$

Martin M, Beume L, Kummerer D, et al. (2015) Differential Roles of Ventral and Dorsal Streams for Conceptual and Production-Related Components of Tool Use in Acute Stroke Patients. Cereb Cortex

Martin M, Nitschke K, Beume L, et al. (2016) Brain activity underlying tool-related and imitative skills after major left hemisphere stroke. Brain

Misaki M, Kim Y, Bandettini PA, Kriegeskorte N (2010) Comparison of multivariate classifiers and response normalizations for pattern-information fMRI. Neuroimage $53: 103-118$

Moll J, de Oliveira-Souza R, Passman LJ, Cunha FC, Souza-Lima F, Andreiuolo PA (2000) Functional MRI correlates of real and imagined tool-use pantomimes. Neurology $54: 1331-1336$

Mruczek RE, von Loga IS, Kastner S (2013) The representation of tool and non-tool object information in the human intraparietal sulcus. J Neurophysiol 109:2883-2896 
Mur M, Bandettini PA, Kriegeskorte N (2009) Revealing representational content with pattern-information fMRI - an introductory guide. Social Cognitive and Affective Neuroscience 4:101-109

Murata A, Gallese V, Luppino G, Kaseda M, Sakata H (2000) Selectivity for the shape, size, and orientation of objects for grasping in neurons of monkey parietal area AIP. J Neurophysiol 83:2580-2601

Noppeney U, Price CJ, Penny WD, Friston KJ (2006) Two distinct neural mechanisms for category-selective responses. Cerebral Cortex 16:437-445

Norman KA, Polyn SM, Detre GJ, Haxby JV (2006) Beyond mind-reading: multi-voxel pattern analysis of fMRI data. Trends Cogn Sci 10:424-430

Ogawa K, Imamizu H (2013) Human sensorimotor cortex represents conflicting visuomotor mappings. Journal of Neuroscience 33:6412-6422

Ogawa K, Inui T (2011) Neural representation of observed actions in the parietal and premotor cortex. Neuroimage 56:728-735

Ogawa K, Inui T (2012) Reference frame of human medial intraparietal cortex in visually guided movements. Journal of Cognitive Neuroscience 24:171-182

Oldfield RC (1971) The assessment and analysis of handedness: the Edinburgh inventory. Neuropsychologia 9:97-113

Oosterhof NN, Tipper SP, Downing PE (2012) Visuo-motor imagery of specific manual actions: a multi-variate pattern analysis fMRI study. Neuroimage 63:262-271

Peeters RR, Rizzolatti G, Orban GA (2013) Functional properties of the left parietal tool use region. Neuroimage 78:83-93

Rice NJ, Valyear KF, Goodale MA, Milner AD, Culham JC (2007) Orientation sensitivity 
to graspable objects: an fMRI adaptation study. Neuroimage 36 Suppl 2:T87-93

Rizzolatti G, Matelli M (2003) Two different streams form the dorsal visual system: anatomy and functions. Exp Brain Res 153:146-157

Rumiati RI, Weiss PH, Shallice T, Ottoboni G, Noth J, Zilles K, Fink GR (2004) Neural basis of pantomiming the use of visually presented objects. Neuroimage $21: 1224-1231$

Tucker M, Ellis R (1998) On the relations between seen objects and components of potential actions. J Exp Psychol Hum Percept Perform 24:830-846

Tunik E, Rice NJ, Hamilton A, Grafton ST (2007) Beyond grasping: representation of action in human anterior intraparietal sulcus. Neuroimage 36 Suppl 2:T77-86

Valyear KF, Cavina-Pratesi C, Stiglick AJ, Culham JC (2007) Does tool-related fMRI activity within the intraparietal sulcus reflect the plan to grasp? Neuroimage 36 Suppl 2:T94-T108

Valyear KF, Culham JC, Sharif N, Westwood D, Goodale MA (2006) A double dissociation between sensitivity to changes in object identity and object orientation in the ventral and dorsal visual streams: a human fMRI study. Neuropsychologia 44:218-228

Vingerhoets G, Acke F, Vandemaele P, Achten E (2009) Tool responsive regions in the posterior parietal cortex: Effect of differences in motor goal and target object during imagined transitive movements. Neuroimage 47:1832-1843 correlates of pantomiming familiar and unfamiliar tools: action semantics versus mechanical problem solving? Hum Brain Mapp 32:905-918

Weiss PH, Achilles EI, Moos K, Hesse MD, Sparing R, Fink GR (2013) Transcranial direct 
current stimulation (tDCS) of left parietal cortex facilitates gesture processing in healthy subjects. J Neurosci 33:19205-19211

Wurm MF, Lingnau A (2015) Decoding actions at different levels of abstraction. J Neurosci $35: 7727-7735$ 


\section{Tables}

Table 1: Anatomical regions, peak voxel coordinates and t-values of observed activation for both hands.

\begin{tabular}{lrrrrr}
\hline Anatomic region & voxels & \multicolumn{3}{c}{ MNI coordinates } & \multirow{2}{*}{-value } \\
\cline { 3 - 5 } & & $\mathrm{x}$ & $\mathrm{y}$ & $\mathrm{Z}$ & \\
\cline { 3 - 6 } & & & & & \\
L inferior occipital gyrus & 2234 & -21 & -94 & 2 & 15.89 \\
$\quad$ L fusiform gyrus & & -39 & -76 & -16 & 12.04 \\
$\quad$ R fusiform gyrus & & 36 & -55 & -25 & 11.68 \\
$\quad$ R inferior occipital gyrus & & 27 & -91 & -1 & 8.16 \\
R pallidum & 218 & 18 & -7 & -4 & 10.44 \\
$\quad$ R putamen & & 24 & -4 & 11 & 7.14 \\
L pallidum & 257 & -24 & -13 & -4 & 9.90 \\
$\quad$ L putamen & & -21 & -1 & 8 & 9.28 \\
L/R supplementary motor area & 196 & 0 & -1 & 65 & 8.54 \\
R cerebellum & 137 & 30 & -55 & -46 & 8.44 \\
L cerebellum & 78 & -33 & -52 & -49 & 8.36 \\
L precentral gyrus & 34 & -18 & -16 & 59 & 8.02 \\
L precentral gyrus & 67 & -39 & -4 & 56 & 7.43 \\
R precentral gyrus & 146 & 45 & 2 & 50 & 7.33 \\
L precentral gyrus & 49 & -30 & -22 & 59 & 6.38 \\
R central operculum & 43 & 45 & -1 & 5 & 6.38 \\
L supramarginal gyrus & 30 & -48 & -34 & 32 & 6.35 \\
L central operculum & 52 & -36 & 5 & 5 & 5.36 \\
L superior parietal lobule & 30 & -36 & -40 & 47 & 5.27 \\
& & & & & \\
\hline
\end{tabular}

MNI, Montreal Neurological Institute; L, left hemisphere; R, right hemisphere. 
Table 2: Anatomical regions, peak voxel coordinates and t-values of observed activation for right versus left hand.

\begin{tabular}{|c|c|c|c|c|c|}
\hline \multirow[t]{2}{*}{ Anatomic region } & \multirow[t]{2}{*}{ voxels } & \multicolumn{3}{|c|}{ MNI coordinates } & \multirow[t]{2}{*}{$t$-value } \\
\hline & & $\mathrm{X}$ & $\mathrm{y}$ & $\mathrm{Z}$ & \\
\hline \multicolumn{6}{|l|}{ Right hand > Left hand } \\
\hline L precentral gyrus & 691 & -33 & -25 & 59 & 12.9 \\
\hline $\mathrm{R}$ cerebellum & 1373 & 30 & -46 & -25 & 8.84 \\
\hline $\mathrm{R}$ middle occipital gyrus & & 36 & -85 & 17 & 8.43 \\
\hline L inferior occipital gyrus & & -21 & -82 & -1 & 8.36 \\
\hline L putamen & 247 & -30 & -13 & -1 & 7.58 \\
\hline L superior parietal lobule & 27 & -24 & -61 & 59 & 6.41 \\
\hline \multicolumn{6}{|l|}{ Left hand > Right hand } \\
\hline L cerebellum & 414 & -3 & -67 & -16 & 14.48 \\
\hline R precentral gyrus & 664 & 36 & -19 & 50 & 9.73 \\
\hline $\mathrm{R}$ superior parietal lobule & & 21 & -46 & 59 & 8.98 \\
\hline R putamen & 167 & 15 & -22 & 5 & 8.04 \\
\hline R supplementary motor area & 30 & 6 & -13 & 50 & 3.98 \\
\hline
\end{tabular}

MNI, Montreal Neurological Institute; L, left hemisphere; R, right hemisphere. 
Table 3: Anatomical regions, peak voxel coordinates and t-values of the MVPA results

\begin{tabular}{lcl}
\hline Anatomic region & voxels $\frac{\text { MNI coordinates }}{n}$ & x -value \\
& & $\mathrm{y} \quad \mathrm{z}$
\end{tabular}

Right hand decoding

L fusiform gyrus

$\begin{array}{rrrrr}282 & -21 & -88 & -7 & 10.90 \\ 450 & -27 & -49 & 59 & 10.00 \\ & -30 & -22 & 65 & 9.38 \\ 94 & 36 & -28 & 38 & 7.90 \\ 166 & 18 & -79 & -4 & 7.36 \\ 59 & 30 & -58 & 53 & 6.56 \\ 17 & -9 & -4 & 59 & 5.84 \\ 19 & -9 & -16 & 50 & 5.59 \\ 15 & 27 & -49 & -22 & 5.22 \\ 12 & -12 & -25 & 44 & 4.52 \\ 12 & -54 & -58 & 2 & 4.25 \\ 12 & 36 & -22 & 56 & 4.04\end{array}$

Left hand decoding

R fusiform gyrus

L postcentral gyrus/aIPS

$\begin{array}{lllll}44 & 21 & -85 & -7 & 9.28\end{array}$

$\mathrm{R}$ precentral gyrus

$\begin{array}{lllll}73 & -21 & -43 & 68 & 7.66\end{array}$

L fusiform gyrus

$\begin{array}{llll}142 & 24 & -19 & 53\end{array}$

6.75

$\begin{array}{llll}24 & -18 & -88 & -7\end{array}$

6.03

Cross decoding between hands

L aIPS

$\begin{array}{llll}27 & -48 & -31 & 50\end{array}$

8.06

MNI, Montreal Neurological Institute; aIPS, anterior intraparietal sulcus; L, left hemisphere; $\mathrm{R}$, right hemisphere. 


\section{Figures}

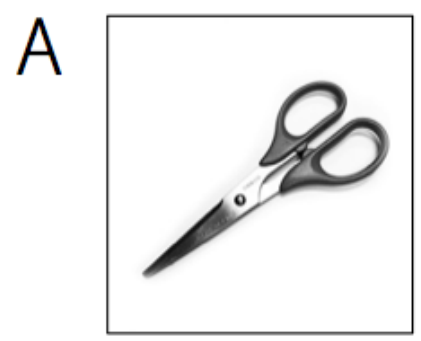

scissors

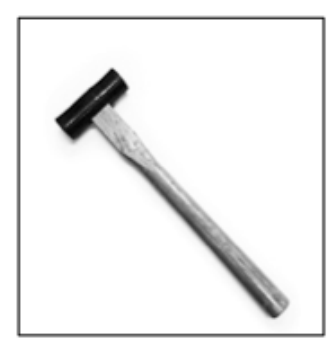

hammer

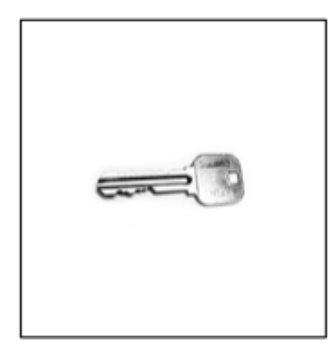

key

B
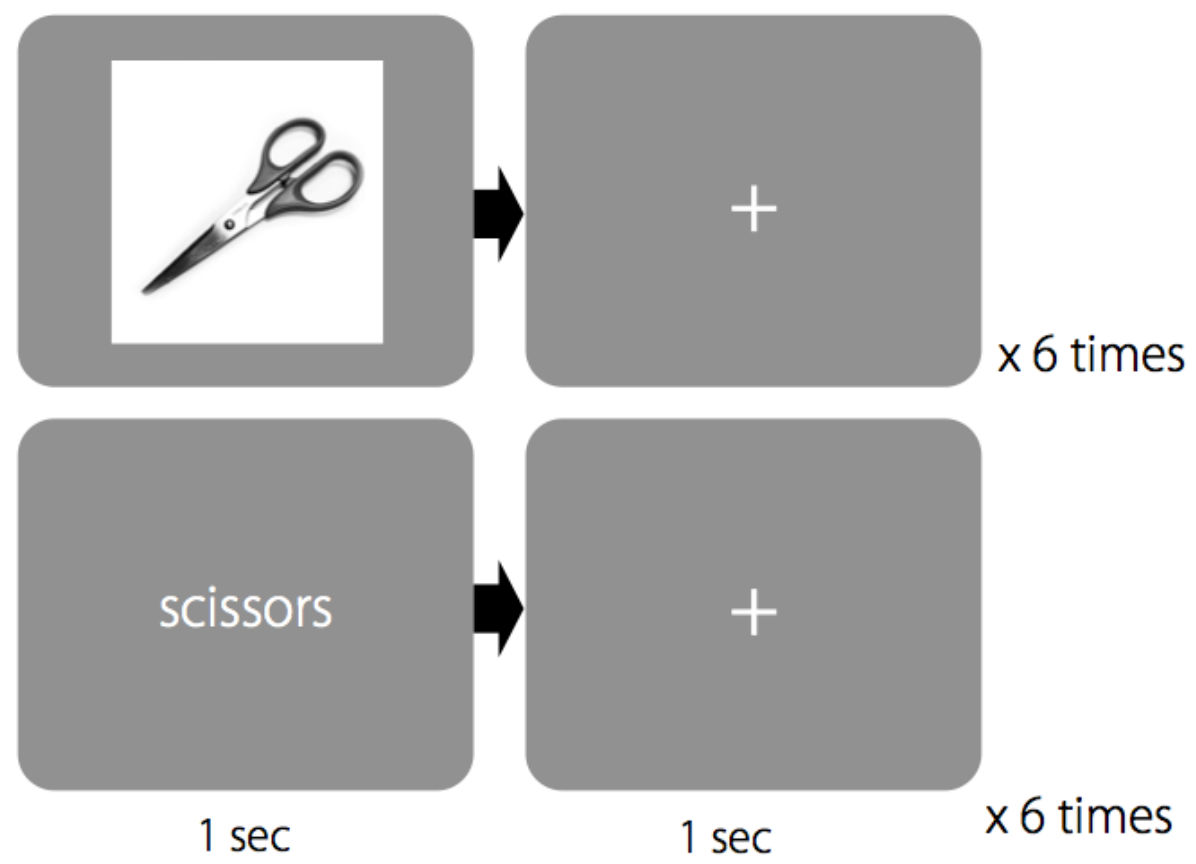

Figure 1:

A) The images of three types of tools used in this experiment. B) The time course of the experiment. Within each task block of $12 \mathrm{~s}$, the grayscale pictures (upper row) or written letters (lower row) of one of the three tools (scissors, hammer, or key) were presented to the participants for $1 \mathrm{~s}$, which was interleaved with fixation for $1 \mathrm{~s}$. The participants were asked to make pantomimes of the use of the displayed tool once for every $2 \mathrm{~s}$, immediately following each stimulus display. The participants used their right or left hands for the picture or word displays, respectively. 


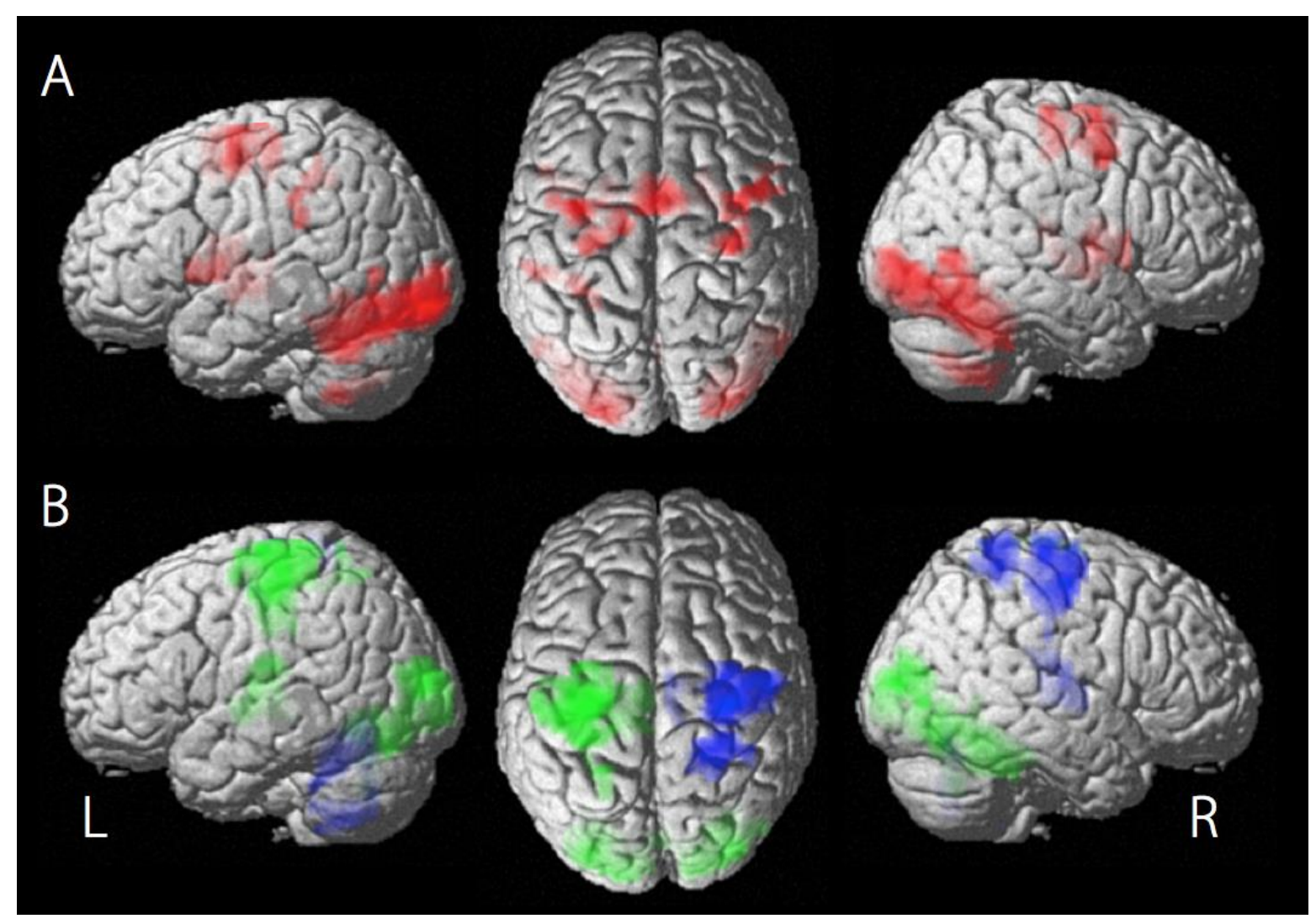

Figure 2:

5 fMRI univariate analysis results. A) Regions activated by all three types of pantomimes, collapsed between both hands. The coordinates of the activated foci are reported in Table 1. B) In green: regions showing a significantly greater activity in the right-hand with pictures condition than in the left-hand with letters condition. In blue: regions showing significantly greater activities in the left-hand with letters condition than in the right-hand with pictures condition. The activity was thresholded at $p<0.001$ uncorrected at the voxel level and at $p$ $<0.05 \mathrm{FWE}$ corrected at the cluster level. The coordinates of the activated foci are reported in Table 2. L, left hemisphere; R, right hemisphere. 


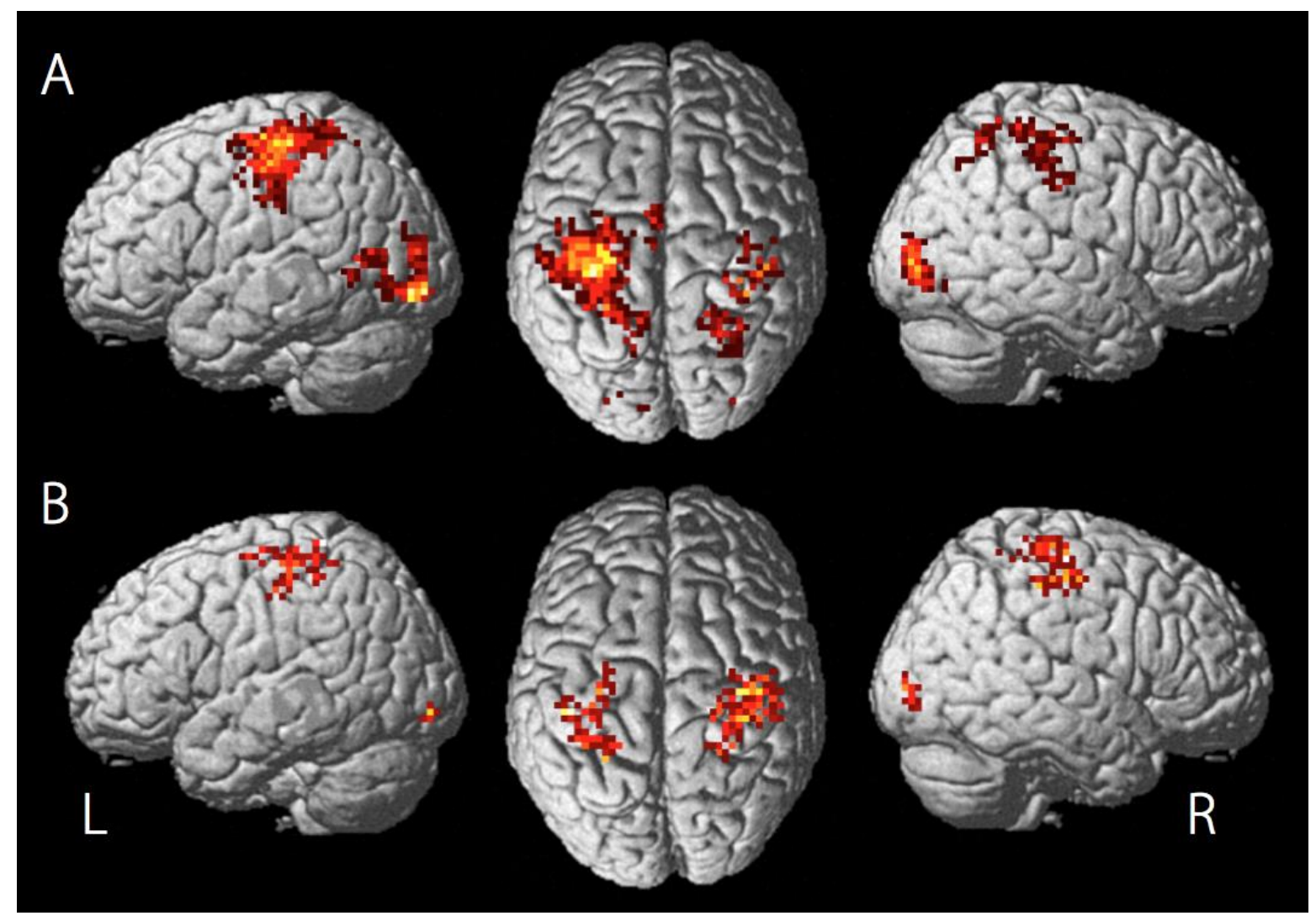

\section{Figure 3:}

5 MVPA searchlight results with hand-dependent classification. A) Regions with significantly above-chance accuracy for pantomimes with the right hand with pictures condition. B) Regions with significantly above-chance accuracy for pantomimes with the left hand with letters condition. The results were thresholded at $p<0.005$ uncorrected at the voxel level and at $p<0.05$ FWE corrected at the cluster level. The coordinates of the observed foci are reported in Table 3. L, left hemisphere; $\mathrm{R}$, right hemisphere. 


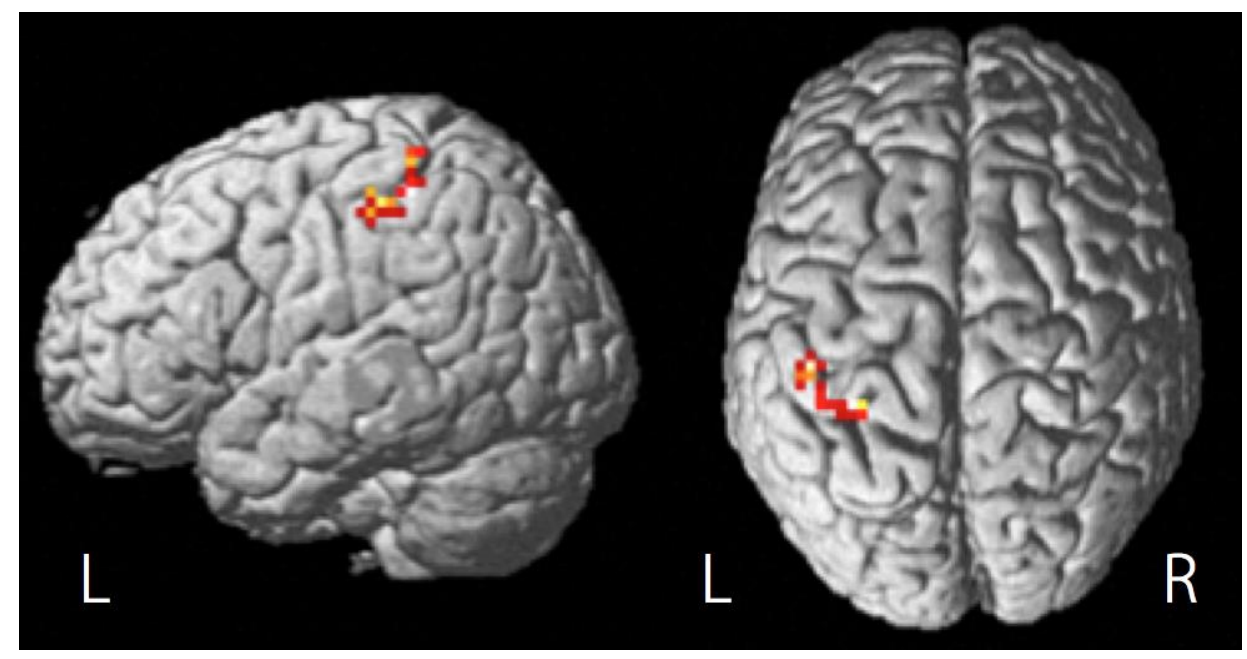

\section{Figure 4:}

MVPA searchlight results with hand-independent cross-classification between the right $5 \quad$ hand with pictures and left hand with letters conditions. The results were thresholded at $p<$ 0.001 uncorrected at the voxel level and at $p<0.05$ FWE corrected at the cluster level. The coordinates of the observed region is reported in Table 3. L, left hemisphere; R, right hemisphere. 


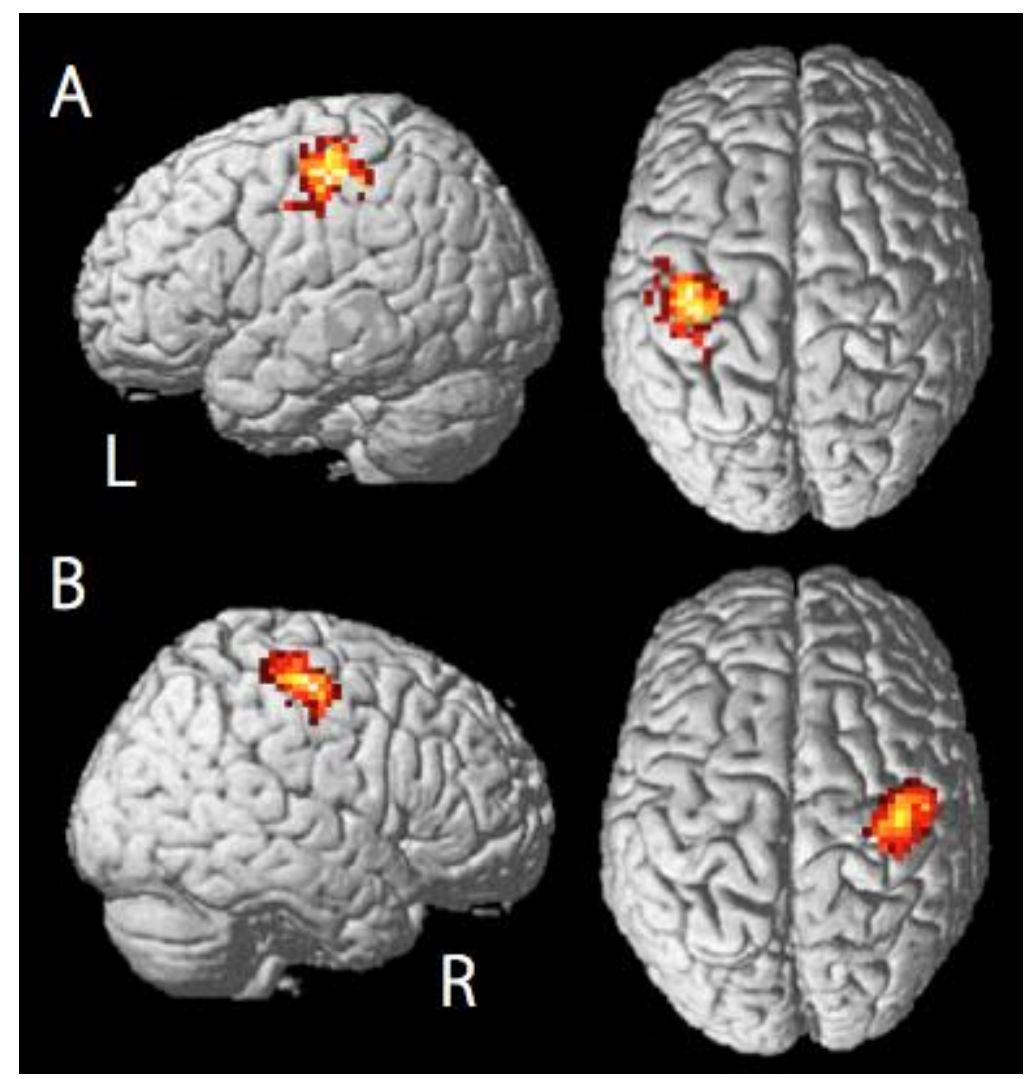

\section{Figure 5:}

MVPA searchlight results with hand-dependent classification for finger tapping. A) Regions

5 with significantly above-chance accuracy for finger tapping with the right hand. B) Regions with significantly above-chance accuracy for finger tapping with the left hand. The results were thresholded at $p<0.005$ uncorrected at the voxel level and at $p<0.05$ FWE corrected at the cluster level. L, left hemisphere; R, right hemisphere. 